\title{
Fli-1 promotes metastasis by regulating MMP2 signaling in hepatocellular carcinoma
}

\author{
HUILING WANG ${ }^{1,2}$, YINGLIANG OU ${ }^{2}$, JINRUI OU ${ }^{2}$ and ZHIXIANG JIAN ${ }^{1,2}$ \\ ${ }^{1}$ Southern Medical University, Guangzhou, Guangdong 510515; ${ }^{2}$ Department of General Surgery, \\ Guangdong General Hospital, Guangdong Academy of Medical Sciences, Guangzhou, Guangdong 510080, P.R. China
}

Received June 27, 2017; Accepted November 3, 2017

DOI: $10.3892 / \mathrm{mmr} .2017 .8047$

\begin{abstract}
Friend leukemia virus integration 1 (Fli-1) is a newly identified ETS protein, and has critical roles in many malignancies. However, the physiological characters and potential mechanisms of Fli-1 in hepatocellular carcinoma (HCC) progression remains unclear. In the present study, Fli-1 was highly expressed in HCC samples and tumor cell lines. knockdown of Fli-1 with small interfering (si)RNAs significantly reduced the colony formation and metastasis capacity of HCC cell lines in vitro. Subsequent investigation identified that Fli-1 functioned as an oncogene in HCC carcinogenesis and it exerted its promoting metastatic effect primarily by modulating the matrix metalloproteinase (MMP)2 signaling pathway. Collectively, these data provide a novel insight into the mechanism of Fli-1/MMP2 signaling pathway in the pathogenesis of HCC, and Fli-1 may serve as a novel therapeutic target for HCC.
\end{abstract}

\section{Introduction}

Hepatocellular carcinoma (HCC) is the third most common cancer-caused death in the worldwild (1). Despite significant therapeutic improvement in clinical treatment, long-term therapeutic efficacy of HCC patients remains poor (2), mainly because of regional invasion and frequent metastasis. However, mechanisms underlying HCC metastasis are not clearly understand, thus it is urgent to identify key biochemical pathways in metastasis to provide new therapeutic targets for HCC.

E26 transformation-specific (ETS) represent a family of highly conserved transcription factors that regulate diverse cellular processes, including proliferation, apoptosis and migration (3). Previous studies have proved that aberrant

Correspondence to: Professor Zhixiang Jian, Department of General Surgery, Guangdong General Hospital, Guangdong Academy of Medical Sciences, 106 Zhongshan Er Road, Yuexiu, Guangzhou, Guangdong 510080, P.R. China

E-mail: 316168299@qq.com

Key words: friend leukemia virus integration 1, hepatocellular carcinoma, metastasis, matrix metalloproteinase 2 expression of ETS was involved in various cancers and played critical roles in regulation of tumorigenesis. Enhanced expression of ETS1 and ETS2 contribute to malignant progression in breast cancer $(4,5)$. In addition, ETS1 promotes invasive potential and lymph node metastasis in human oral squamous cell carcinoma (6).

Friend leukemia virus integration 1 (Fli-1), a newly identified member of the ETS family, was first isolated in friend murine leukemia virus (F-MuLV) caused erythroleukemia (7). It emerged as a transcriptional activator or repressor in embryonic development, hematopoiesis, aberrant vasculogenesis (8-10).

Emerging evidences have shown that dysregulation of Fli-1 is a feature of many malignancies, such as breast cancer (11), melanoma (12), Ewing's sarcoma $(13,14)$, Nasopharyngeal carcinoma (15). It modulates different pathological process and functions as an oncogene or tumor suppressor in different tissue types. However, studies on elucidating the potential role of Fli-1 in HCC progression are rare. In this study, we studied the expression level of Fli-1 in HCC. Furthermore, we examined the effects of Fli-1 on HCC tumorigenesis and the underlying mechanisms.

\section{Materials and methods}

Patients and tissue specimens. A total of 50 matched specimens of cancer-adjacent tissue and HCC tissue, including

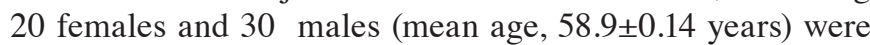
obtained from patients who underwent surgical resection at the Guangdong General Hospital (Guangzhou, China). Each matched cancer-adjacent tissue was obtained at least $10 \mathrm{~cm}$ from the tumor margin. All the samples enrolled in this study were histologically confirmed by pathologists, and none of the patients had received chemotherapy or radiotherapy before the surgery.

This study was approved by the Research Ethics Committee of Guangdong General Hospital and written informed consent was obtained from each patient.

Cell lines. The human HCC cell line BEL7402, PLC/PRF/5, SMMC7721, MHCC97H and a normal human liver cell line HL-7702 were obtained from the Cell Bank of the Chinese Academy of Science (Shanghai, China) and maintained in DMEM (Gibco, Grand Island, NY, USA) supplemented with 
$10 \%$ fetal bovine serum (Gibco) in a humidified chamber with $5 \%(\mathrm{v} / \mathrm{v}) \mathrm{CO}_{2}$ at $37^{\circ} \mathrm{C}$.

Construction of plasmid or small interfering (si)RNA sequences and transfection. The full-length Fli-1 cDNA was amplified and cloned into the pcDNA3.1 expression vector (Cyagen Biosciences Inc., Guangzhou, China). The expression plasmid and control plasmid were transfected respectively into SMMC7721 and MHCC97H cells with Lipofectamine 2000 (Invitrogen, Carlsbad, CA, USA) following the protocol of the manufacturer.

Primer sequences used for PCR were: Sense, 5'-AAA CGAGGGAAATGGGAG-3' and antisense, 5'-TACCAACGG TGTCAACCTG-3'. The sequences of siFli-1 were as follows: siFli-1 \#1, 5'-AGGAGUGGAUCAAUCAGCCAGUGAG-3' and siFli-1 \#2, 5'-GGGAAAGUUCACUGUUGGCCUA UAA-3'. Negative control (siNC) was: 5'-CAGUACUUUUGUG UAGUACAA-3'. All sequences were synthesized by Invitrogen. The transfection of siFli-1 and siNC was performed with RNAiMAX transfection reagent.

RNA extraction and quantitative PCR. Total RNA extractions from cells and tissue specimens were performed using the TRIzol reagent (Invitrogen) according to the manufacturer's instructions. Then the RNA was reversely transcribed to cDNA with the cDNA synthesis kit (Takara Bio, Inc., Otsu, Japan) using $1 \mu \mathrm{g}$ RNA as template. Real-time PCR primers were synthesized by Invitrogen. GAPDH was used as the reference for mRNAs. Specific primers used were: Fli-1 forward, 5'-CAGTCGCCTAGCCAACCCTG-3' and reverse, 5'-GCA ATGCCGTGGAAGTCAAAT-3'; GAPDH forward, 5'-GCA CCGTCAAGGCTGAGAAC-3' and reverse, 5'-TGGTGAAGA CGCCAGTGGA-3'.

Colony formation assay. For colony formation assays, 1,000 cells/well were seeded onto 6 -well plates and incubated at $37^{\circ} \mathrm{C}$ in a humidified incubator for 2 weeks. When the colonies were visible, fixed with $4 \%$ paraformaldehyde and stained with $1 \%$ crystal violet. Finally, the numbers of colonies were calculated.

Migration and invasion assay. Cell migration and invasion assay were performed with Transwell chambers (Corning Incorporated, Corning, NY, USA). For invasion assay, the upper chamber was pre-coated with Matrigel. Cells [5×104 (for migration assay) or $1 \times 10^{5}$ (for invasion assay)] were resuspended in serum-free DMEM and $200 \mu \mathrm{l}$ was added to the upper chamber. DMEM $(600 \mu \mathrm{l})$ supplemented with $10 \%$ FBS was added to the lower chamber. After $12 \mathrm{~h}$ incubation at $37^{\circ} \mathrm{C}$, the membrane was fixed with methanol and stained by crystal violet, the number of migrating cells was counted under the microscope.

Wound-healing assay. For wound healing assay, tumor cells were seeded in 12-well plates (12,000 cells per well), scratch wounds were made using a sterilized $200 \mu$ l plastic pipette tip in the middle of the cell monolayer. The width of wounds was measured under the microscope and wound-healing percentage was calculated.

Western blotting. Total protein was extracted using the lysis buffer (Beyotime Institute of Biotechnology, Haimen, China).
Next, proteins were quantified with the Bradford assay (Bio-Rad Laboratories, Inc., Hercules, CA, USA), $20 \mu \mathrm{g}$ of different proteins were subjected to $12 \%$ sodium dodecylsulfate polyacrylamide gel electrophoresis (SDS-PAGE) and transferred to the polyvinylidene difluoride (PVDF) membranes (EMD Millipore, Billerica, MA, USA). After blocking in 5\% skim milk at room temperature for $2 \mathrm{~h}$, the membranes were incubated with primary antibodies overnight at $4^{\circ} \mathrm{C}$ and followed by matched horseradish peroxidase (HRP)-conjugated second antibody at room temperature for $1 \mathrm{~h}$. Protein staining was measured by chemiluminescence (GE Healthcare Life Sciences, Little Chalfont, UK). The antibodies used were as follows: Rabbit anti-Fli-1 pAb (11347-1-AP; 1:1,000; ProteinTech Group, Inc., Chicago, IL, USA), rabbit anti-matrix metalloproteinase (MMP)2 mAb (\#4022; 1:1,000; Cell Signaling Technology, Inc., Danvers, MA, USA), GAPDH rabbit mAb (\#2118; 1:2,000; Cell Signaling Technology, Inc.) was used as an internal control protein.

Statistical analysis. Statistically analysis between the two groups was calculated using Student's t-test and one-way ANOVA followed by Scheffe test (post hoc) was used for multiple groups. Correlations between FLI-1 expression level and clinicopathological data were analyzed using Chi-square test. Data are shown as means \pm standard deviation (SD) from at least three independent experiments, $\mathrm{P}<0.05$ was considered statistically significant.

\section{Results}

Fli-1 was overexpressed in human HCC tissues and cell lines and was associated with metastasis. Abnormally Fli-1 expression has been reported in many malignancies, but whether Fli-1 deregulation also exists in HCC remains unknown. Therefore, quantitative real-time PCR (qRT-PCR) analysis was first carried out to examine the expression of Fli-1 in 50 matched HCC and cancer-adjacent tissues. As shown in Fig. 1A, Fli-1 mRNA level was dramatically elevated in the majority of HCC tissues compared to cancer-adjacent tissues $(\mathrm{P}<0.001)$. We next determined the expression pattern of Fli-1 in 5 paired HCC samples and adjacent normal tissues by western blotting (Fig. 1B). Western blot analysis also showed overexpression of Fli-1 in HCC tissues examined, compared with matched noncancerous samples. We next examined the correlation between FLI-1 mRNA expression and clinicopathologic characteristics in $50 \mathrm{HCC}$ patients, the results were shown in Table I. However, no statistically significant association were found between FLI-1 expression and clinicopathologic features except for metastasis.

In order to further validate our finding, we then confirmed the expression of Fli-1 in HCC cell lines. Western blot analysis showed a significant upregulation of Fli-1 in all HCC cell lines, compared with normal liver cell line (Fig. 1C). Besides, RT-PCR analysis was performed to detect whether high expression of Fli-1 was also occurred at transcriptional level. Data in Fig. 1D showed that Fli-1 mRNA expression also elevated in all HCC cell lines compared with normal liver cell line.

Additionally, among the 4 HCC cell lines, Fli-1 expression was much higher in SMMC7721 cells than that in other 
Table I. Correlation between Fli-1 level and clinicopathologic characteristics.

\begin{tabular}{|c|c|c|c|c|}
\hline \multirow[b]{2}{*}{ Characteristics } & \multirow{2}{*}{$\begin{array}{l}\text { No. of } \\
\text { patients } \\
(\mathrm{n}=50)\end{array}$} & \multicolumn{2}{|c|}{$\begin{array}{l}\text { Fli- } 1 \text { expression } \\
\quad(\text { mean) }\end{array}$} & \multirow[b]{2}{*}{ P-value } \\
\hline & & $\begin{array}{l}\text { Low } \\
(\mathrm{n}=12)\end{array}$ & $\begin{array}{l}\text { High } \\
(\mathrm{n}=38)\end{array}$ & \\
\hline Sex & & & & 0.362 \\
\hline Male & 40 & 8 & 32 & \\
\hline Female & 10 & 4 & 6 & \\
\hline Age (years) & & & & 0.639 \\
\hline$\leq 50$ & 30 & 6 & 24 & \\
\hline$>50$ & 20 & 6 & 14 & \\
\hline $\operatorname{AFP}(\mathrm{ng} / \mathrm{ml})$ & & & & 0.296 \\
\hline$\leq 400$ & 37 & 7 & 30 & \\
\hline$>400$ & 13 & 5 & 8 & \\
\hline $\mathrm{T}$ classification & & & & 0.410 \\
\hline T1-2 & 26 & 5 & 21 & \\
\hline T3-4 & 24 & 7 & 17 & \\
\hline TNM & & & & 0.417 \\
\hline $\mathrm{I}+\mathrm{II}$ & 32 & 6 & 26 & \\
\hline III+IV & 18 & 6 & 12 & \\
\hline Cirrhosis & & & & 0.137 \\
\hline Yes & 39 & 7 & 32 & \\
\hline No & 11 & 5 & 6 & \\
\hline Distant metastasis & & & & 0.028 \\
\hline Yes & 22 & 2 & 20 & \\
\hline No & 28 & 10 & 18 & \\
\hline
\end{tabular}

Fli-1, friend leukemia virus integration 1 .

HCC cell lines, whereas MHCC97H showed less Fli-1 level. Besides, SMMC-7721 and MHCC97H cell lines showed high migratory potential. Thus, SMMC-7721 and MHCC97H cell lines were chosen for subsequent study.

Silence of Fli-1 suppresses clone formation of HCC cells. The above data suggest that Fli-1 deregulation may play a physiological role in HCC tumorigenesis. To ascertain the link between Fli-1 expression and tumor progression, we reduced Fli-1 expression in SMMC7721 cells and enhanced Fli-1 expression in MHCC97H cells. Fig. 2 indicated each individual siRNA (siRNA \#1 or siRNA \#2) markedly reduced Fli-1 mRNA expression, compared with siNC (Fig. 2A). Knockdown efficiency was further verified at the protein level (Fig. 2C). Similarly, Fli-1 level was increased in MHCC97H cells after transfection with pcDNA3.1-Fli-1 plasmid at both mRNA (Fig. 2B) and protein level (Fig. 2D).

Since tumor cells growth and clone formation play crucial roles in tumor metastasis progression (16), clone formation assay was performed to examine the role of Fli-1 in HCC cell growth. Seven days after siRNA transfection, we fixed and stained the colonies. As shown in Fig. 2E, knockdown of Fli-1 sharply suppressed the number and size of colonies, compared

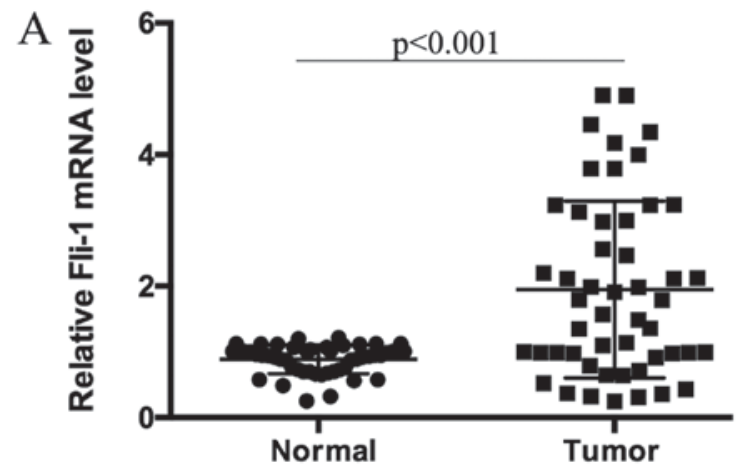

$\begin{array}{llllllllllll}\text { B } & \text { N1 } & \text { T1 } & \text { N2 } & \text { T2 } & \text { N3 } & \text { T3 } & \text { N4 } & \text { T4 } & \text { N5 } & \text { T5 }\end{array}$
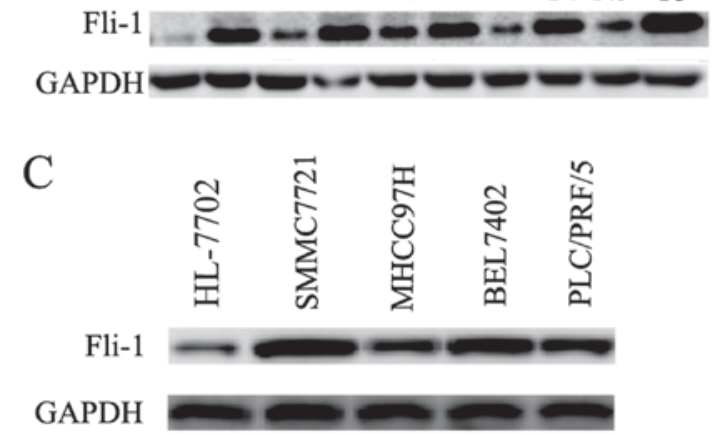

$\mathrm{D}$

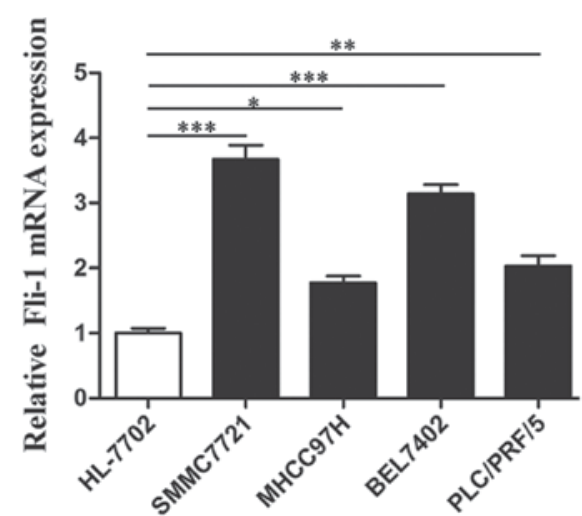

Figure 1. Fli-1 is upregulated in HCC samples and cell lines. (A) The average expression level of Fli-1 was measured in 50 human HCC tissues and matched tumor-adjacent tissues by real-time PCR. (B) Expression of Fli-1 in 5 pairs of matched primary tumors $(\mathrm{T})$ and adjacent non-tumor tissue $(\mathrm{N})$ by western blot analysis. GAPDH was used as loading control. (C) Fli-1 was overexpressed in HCC cell lines at protein level. GAPDH was used as the internal control. (D) Fli-1 was overexpressed in HCC cell lines at mRNA level. Data are shown as mean \pm standard deviation; ${ }^{*} \mathrm{P}<0.05 ;{ }^{* *} \mathrm{P}<0.01 ;{ }^{* * *} \mathrm{P}<0.001$ vs. control. Fli- 1 , friend leukemia virus integration 1; HCC, hepatocellular carcinoma.

with control. As expected, Fli-1 overexpression in MHCC97H cells led to a significant enhancement of clone formation (Fig. 2F).

Knockdown of Fli-1 inhibits HCC cell migration and invasion. To investigate the role of Fli-1 in tumor cells migration, wound-healing assay was performed. We observed that knockdown of Fli-1 expression dramatically inhibited wound closure in SMMC7721 cells (Fig. 3A). Moreover, Transwell assay also demonstrated that the migratory capacity was dramatically decreased in siFli-1-transfected cells (Fig. 3B).

To further confirm this conclusion, we conducted a Transwell assay with Matrigel pre-coated to detect the cell invasion. 
A

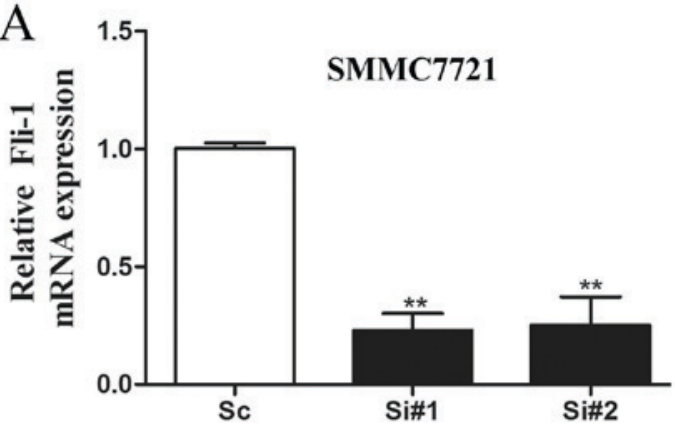

C
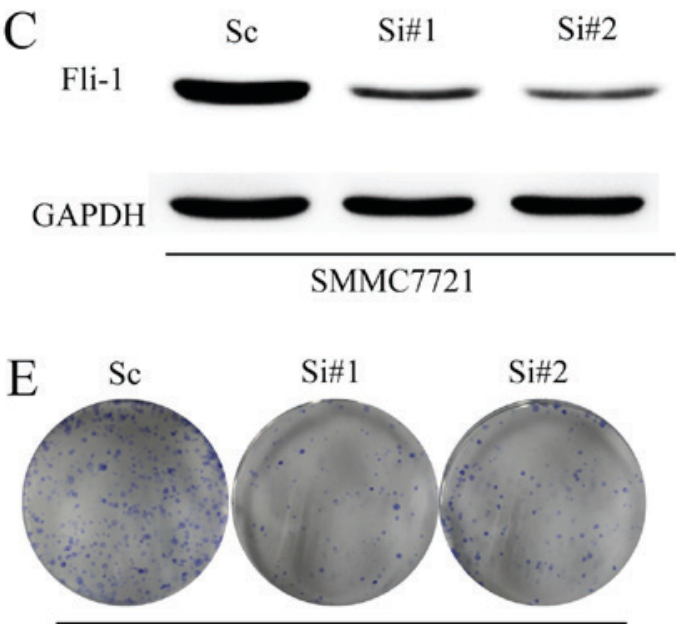

SMMC7721

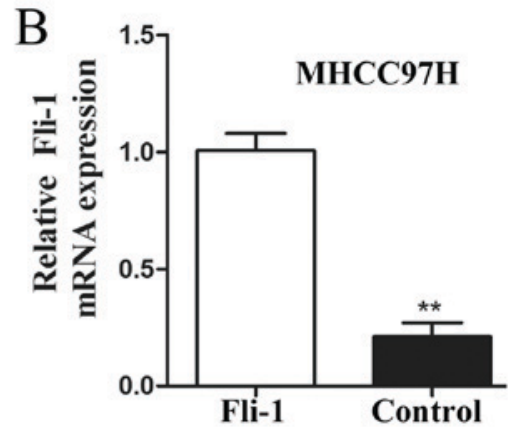

D

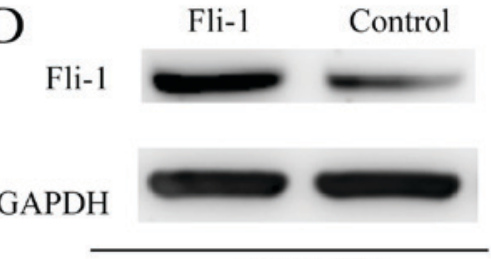

MHCC97H

$\mathrm{F}$

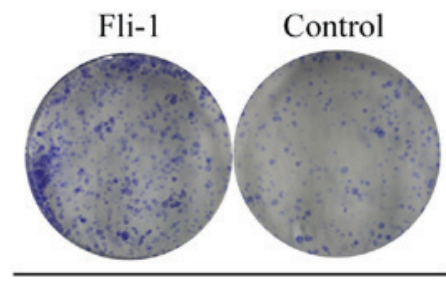

$\mathrm{MHCC} 97 \mathrm{H}$

Figure 2. Inhibition of Fli-1 suppresses clone formation of HCC cells. (A) SMMC7721 cells were transfected with scrambled siRNA (Sc), Fli-1-siRNA\#1 (Si\#1), siRNA\#2 (Si\#2). Fli-1 expression was measured by real-time PCR. Data are shown as mean \pm SD; ${ }^{* *} \mathrm{P}<0.01 \mathrm{vs}$. control. (B) Fli-1 mRNA level in MHCC97H after transfection with pcDNA3.1-Fli-1 plasmid. Data are shown as mean $\pm \mathrm{SD} ;{ }^{* *} \mathrm{P}<0.01$ vs. control. (C) Fli-1 protein level in SMMC7721 cells following transfection with scrambled siRNA (Sc), Fli-1-siRNA\#1 (Si\#1), siRNA\#2 (Si\#2). (D) Fli-1 protein level in MHCC97H after transfection with pcDNA3.1-Fli-1 plasmid. (E) Silence of Fli-1 inhibited colony formation in SMMC7721 cells. (F) Enhanced FLI-1 expression increased colony formation in MHCC97H cells. Representative pictures of clone formation assay as showed. Fli-1, friend leukemia virus integration 1; HCC, hepatocellular carcinoma; siRNA, small interfering RNA; SD, standard deviation.

Compared with the NC group, the number of migrating cells in Fli-1 knockdown group was sharply decreased (Fig. 3C). In contrast, enhanced Fli-1 expression highly promoted MHCC97H cell migration and invasion (Fig. 3D-F).

Overall, these results revealed that Fli-1 significantly promoted cell migration and invasion in vitro.

Decreasing Fli-1 expression alters MMP2 expression. Given that MMPs were closely associated with tumor cells metastasis, We next investigated whether Fli-1 mediated metastasis promoting effect was related to MMPs. MMP2 expression was examined after transfection with siRNA or pcDNA3.1-Fli-1 plasmid. As shown in Fig. 4A, downregulating Fli-1 expression was accompanied by decreased MMP2 mRNA level, as compared to the control group. This result was also reproducible at protein level (Fig. 4B). In the contrast, enhanced MMP2 expression was observed in Fli-1 overexpressed SMMC7721 cells (Fig. 4C and D). Consistently, Fli-1 overexpression obviously increased MMP2 level in MHCC97H cells (Fig. 4E).

\section{Discussion}

Fli-1 is a newly identified ETS family member and localized on human chromosome 11q23 (8). It was first discovered in mouse erythroleukemia, while in human, EWS/Fli-1 fusion oncogene became characteristic of Ewing sarcoma because of a $\mathrm{t}(11 ; 22)$ (q24;q12) chromosomal translocation (13). Fli-1 plays vital roles in various biological processes. Aberrant activation of Fli-1 is associated with inhibition of erythroid differentiation and apoptosis, and leads to an increase in proliferation of erythroblasts (8), whereas loss of Fli-1 could impair vascular homeostasis in endothelial cells (17). Besides, aberrant expression of Fli-1 has been demonstrated in various malignancies, such as breast cancer (11), nasopharyngeal carcinoma (NPC) (15), colon cancer (18) and osteosarcoma (19). High expression of Fli-1 contributes to the metastasis and tumorigenesis of cancer $(11,15)$, while inhibition of Fli-1 leads to growth suppression and apoptosis of colon tumor cells (18), indicating that Fli-1 may play a critical role in tumor progression. However, the physiological function and the underlying mechanism of Fli-1 in HCC tumorigenesis remain unclear.

In the present study, we demonstrated that Fli-1 is upregulated both in HCC cancer cell lines and in primary HCC cancer tissues, suggesting that Fli-1 is involved and may function as an oncogene in HCC progression.

To further elucidate the role of Fli-1 in HCC progression, increase and depletion experiments were respectively conducted in MHCC97H and SMMC7721 cells. Loss of Fli-1 

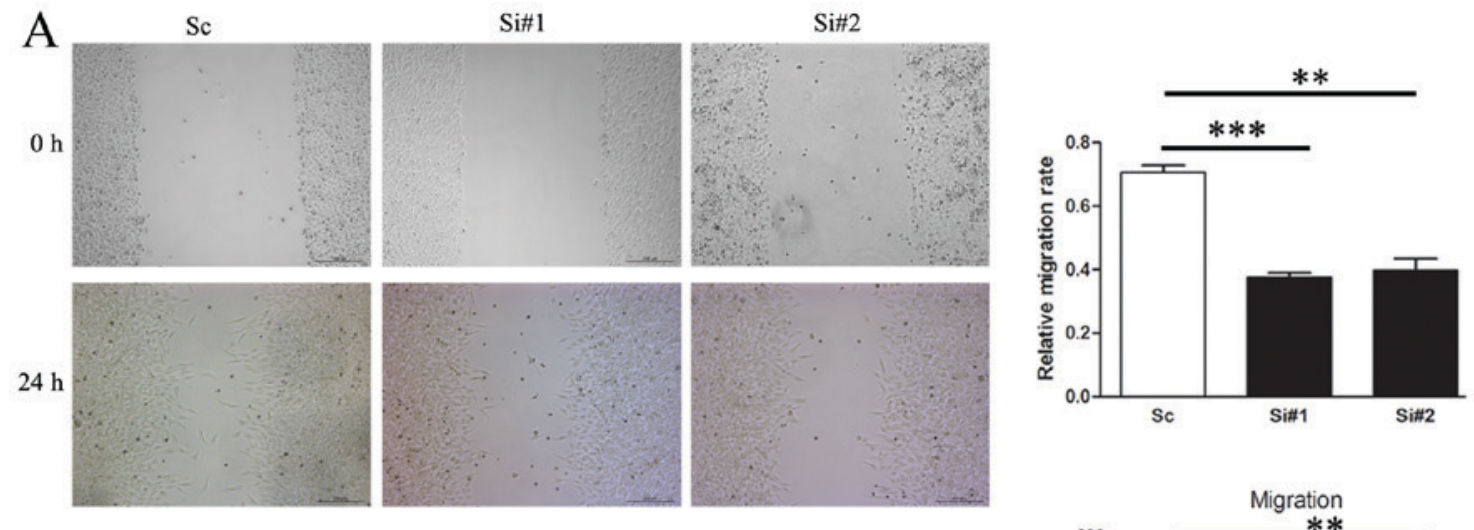

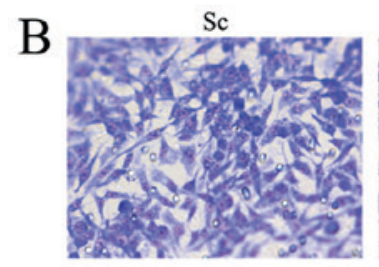

Si\#1

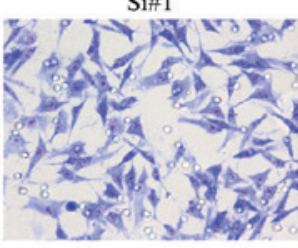

Si\#1

$\mathrm{C}$
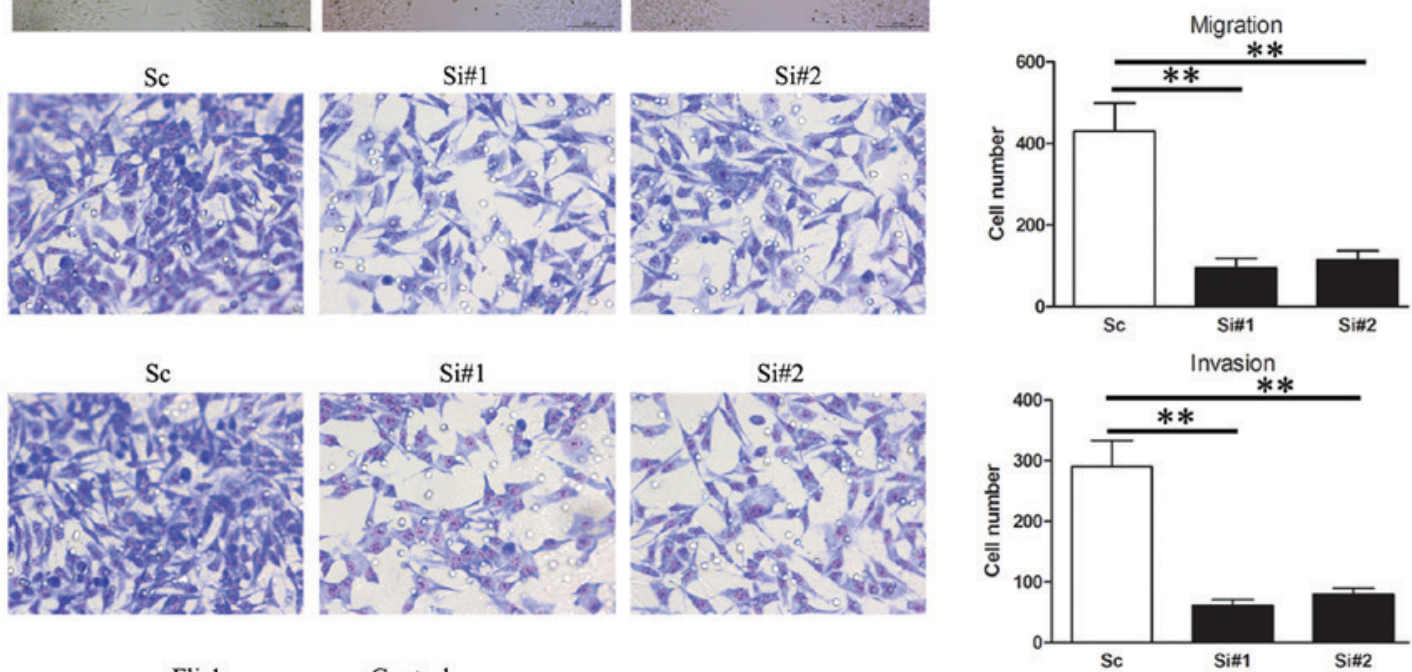

Fli-1

D

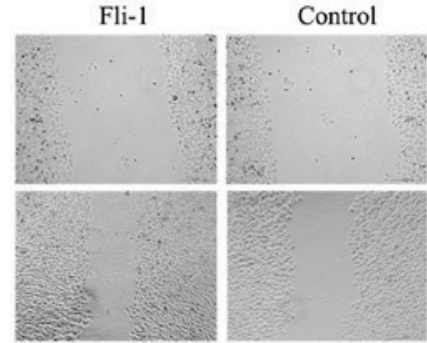

E

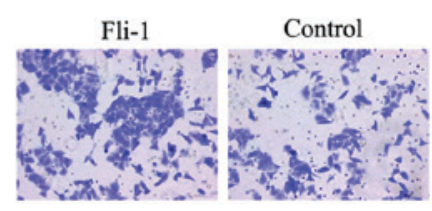

F

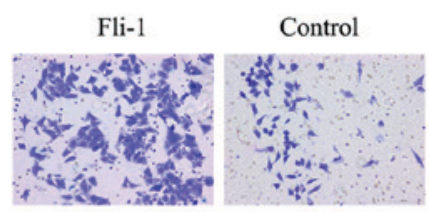

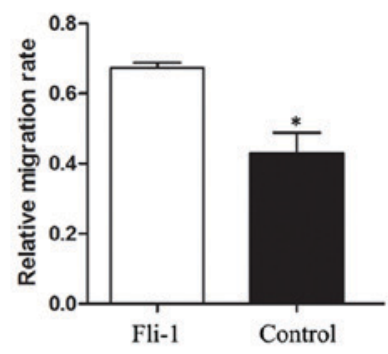
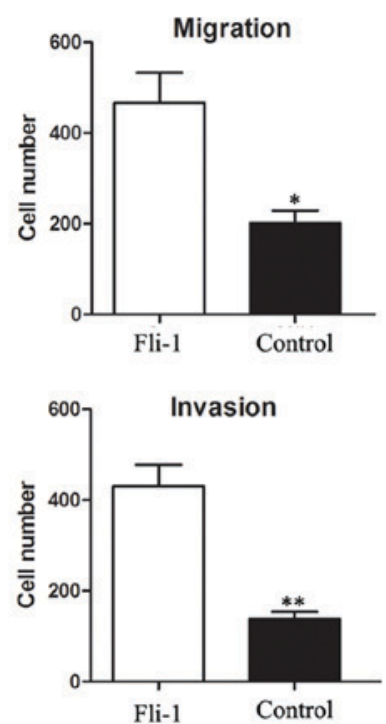

Figure 3. Knockdown of Fli-1 inhibits HCC cell migration and invasion. (A) Wound healing assay was performed in SMMC7721 cells 24 h after transfection with scrambled siRNA (Sc), Fli-1-siRNA\#1 (Si\#1), siRNA\#2 (Si\#2). Data are shown as mean \pm SD; ${ }^{* *} \mathrm{P}<0.01$ and ${ }^{* * *} \mathrm{P}<0.001$ vs. control. (B) Cell migration was further detected with Transwell assays. Data are shown as mean $\pm \mathrm{SD} ;{ }^{* *} \mathrm{P}<0.01$ vs. control. (C) Invasion analysis was evaluated with matrigel pre-coated, the number of migrating cells was counted under the microscope. Data are shown as mean $\pm \mathrm{SD} ;{ }^{* *} \mathrm{P}<0.01$ vs. control. (D) Wound healing assay was conducted in MHCC97H cells after transfection with pcDNA3.1-Fli-1 plasmid. Data are shown as mean \pm SD; ${ }^{*} \mathrm{P}<0.05$ vs. control. (E) Enhanced expression of Fli-1 promoted cell migration in MHCC97H cells. Data are shown as mean $\pm \mathrm{SD} ;{ }^{*} \mathrm{P}<0.05$ vs. control. (F) Fli-1 promoted cell invasion in MHCC97H cells. Data are shown as mean \pm SD; ${ }^{* *} \mathrm{P}<0.01$ vs. control. Fli-1, friend leukemia virus integration 1; HCC, hepatocellular carcinoma; siRNA, small interfering RNA; SD, standard deviation. 
A

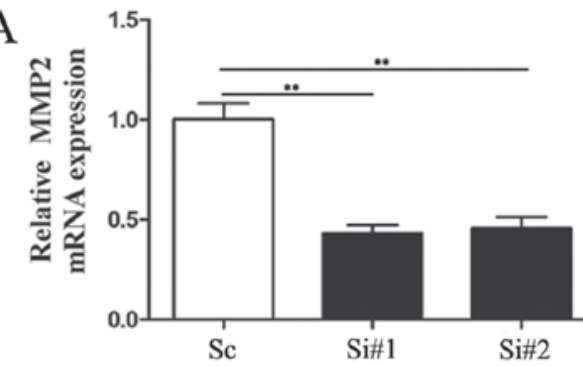

$\mathrm{C}$

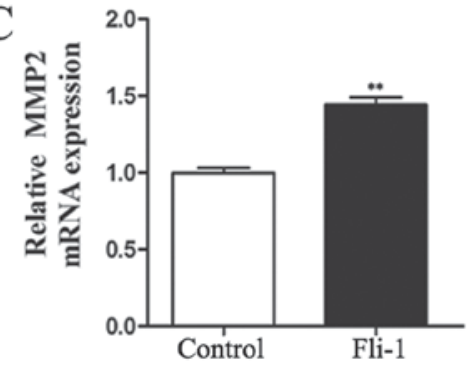

B

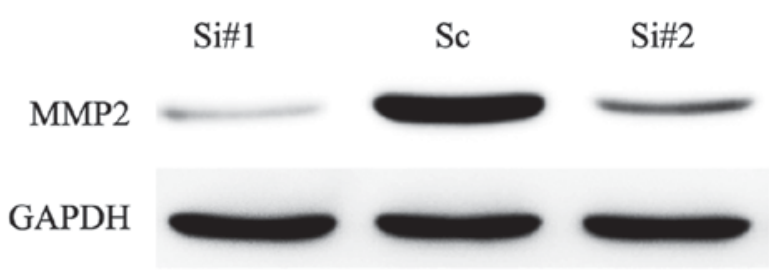

E
$\mathrm{D}$

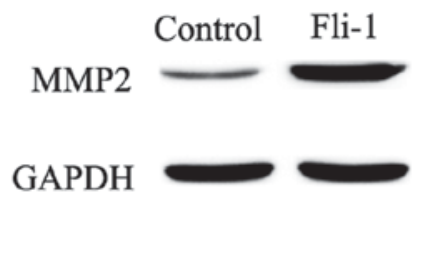

MMP2

Fli-1 Control

\section{GAPDH}

Figure 4. Decreasing Fli-1 expression suppresses MMP2 expression. (A) MMP2 mRNA level in SMMC7721 cells was measured following transfection with scrambled siRNA (Sc), Fli-1-siRNA\#1 (Si\#1), siRNA\#2 (Si\#2). Data are shown as mean \pm SD; ${ }^{* *} \mathrm{P}<0.01$ vs. control. (B) MMP2 expression was further detected at protein level. (C) Enhanced expression of Fli-1 upregulated the expression of MMP2 both at mRNA level. Data are shown as mean \pm standard deviation; ${ }^{* *} \mathrm{P}<0.01$ vs. control. (D) Protein level in SMMC7721 cells, compared with the control group. (E) Fli-1 overexpression increased MMP2 expression in MHCC97H cells. Fli-1, friend leukemia virus integration 1; MMP, matrix metalloproteinase; siRNA, small interfering RNA; SD, standard deviation.

suppresses the invasion and metastasis of SMMC7721 cells in vitro, whereas ectopic overexpression of Fli-1 facilitates metastasis, which is consistent with the effect of Fli-1 in breast cancer and NPC $(11,15)$ and further support our notion that Fli-1 is involved in HCC malignant transformation.

Metastasis is a heterogeneous process including cytoskeletal changes, integrin based adhesion, cell-extracellular matrix (ECM) interactions, localized proteolysis, actin-myosin contractions, and focal contact disassembly (20), in which active angiogenesis and disruption of the ECM were the two crucial steps. Aberrant activation of MMPs have been reported to be involved in the degradation of ECM and the basal membrane, which assists the migration and invasion of tumor. MMPs are a family of ECM proteases $(21,22)$, They can modulate tumor spread and metastasis by facilitating ECM degradation, remodeling ECM, maintaining the tumor micro-environment and generating ECM-linked growth factors. In the MMPs family, MMP2 belongs to a group of gelatinases. It is a key mediator in ECM degradation and is involved in angiogenesis and metastasis in various malignancies $(23,24)$, including $\mathrm{HCC}(25,26)$.

This promoted us to investigate the link of MMP2 with Fli-1 in the metastasis of HCC. Here, we found that knockdown of Fli-1 with siRNAs significantly repressed MMP2 expression, and inhibited invasion and metastasis of tumor cells in vitro. Accordingly, enhanced expression of Fli-1 is associated with increased level of MMP2, accompanied with increased invasion and migration of tumor cells. It is suggested that Fli-1 exerts its promoting metastatic effect in HCC through the activation of MMP2 signaling. However, the potential molecular mechanism of Fli-1 exerts its modulation effect on the expression of MMP2 is still not known, needs to be further explored.

In conclusion, our results demonstrated that high expression of Fli-1 in HCC was associated with tumor metastasis, and involved in the HCC tumor progression. These data suggest that Fli-1 functions as an oncogene in HCC carcinogenesis in vitro, although its anticancer effect needs to be further verified in vivo. Our data provide novel insight into the mechanism of Fli-1/MMP2 pathway in the pathogenesis of HCC. Furthermore, Fli-1 and relevant downstream signaling may serve as new therapeutic targets for HCC.

\section{References}

1. Forner A, Llovet JM and Bruix J: Hepatocellular carcinoma. Lancet 379: 1245-1255, 2012.

2. Feng M, Gao W, Wang R, Chen W, Man YG, Figg WD, Wang XW, Dimitrov DS and Ho M: Therapeutically targeting glypican-3 via a conformation-specific single-domain antibody in hepatocellular carcinoma. Proc Natl Acad Sci USA 110: E1083-E1091, 2013.

3. Seth A and Watson DK: ETS transcription factors and their emerging roles in human cancer. Eur J Cancer 41: 2462-2478, 2005.

4. Buggy Y, Maguire TM, McGreal G, McDermott E, Hill AD, O'Higgins $\mathrm{N}$ and Duffy MJ: Overexpression of the Ets-1 transcription factor in human breast cancer. Br J Cancer 91: 1308-1315, 2004.

5. Buggy Y, Maguire TM, McDermott E, Hill AD, O'Higgins N and Duffy MJ: Ets2 transcription factor in normal and neoplastic human breast tissue. Eur J Cancer 42: 485-491, 2006.

6. Shintani S, Hamakawa H, Nakashiro K, Shirota T, Hatori M, Tanaka M, Kuroshita Y and Kurokawa Y: Friend leukaemia insertion (Fli)-1 is a prediction marker candidate for radiotherapy resistant oral squamous cell carcinoma. Int J Oral Maxillofac Surg 39: 1115-1119, 2010.

7. Ben-David Y, Giddens EB, Letwin K and Bernstein A: Erythroleukemia induction by friend murine leukemia virus: Insertional activation of a new member of the ets gene family, Fli-1, closely linked to c-ets-1. Genes Dev 5: 908-918, 1991.

8. Truong AH and Ben-David Y: The role of Fli-1 in normal cell function and malignant transformation. Oncogene 19: 6482-6489, 2000.

9. Kawada H, Ito T, Pharr PN, Spyropoulos DD, Watson DK and Ogawa M: Defective megakaryopoiesis and abnormal erythroid development in Fli-1 gene-targeted mice. Int J Hematol 73: 463-468, 2001. 
10. Spyropoulos DD, Pharr PN, Lavenburg KR, Jackers P, Papas TS, Ogawa $M$ and Watson DK: Hemorrhage, impaired hematopoiesis, and lethality in mouse embryos carrying a targeted disruption of the Fli1 transcription factor. Mol Cell Biol 20: 5643-5652, 2000.

11. Song W, Li W, Li L, Zhang S, Yan X, Wen X, Zhang X, Tian H, $\mathrm{Li} \mathrm{A}, \mathrm{Hu} \mathrm{JF}$ and Cui J: Friend leukemia virus integration 1 activates the Rho GTPase pathway and is associated with metastasis in breast cancer. Oncotarget 6: 23764-23775, 2015.

12. Torlakovic EE, Slipicevic A, Flørenes VA, Chibbar R, DeCoteau JF and Bilalovic N: Fli-1 expression in malignant melanoma. Histol Histopathol 23: 1309-1314, 2008.

13. Ladanyi M: EWS-FLI1 and Ewing's sarcoma: Recent molecular data and new insights. Cancer Biol Ther 1: 330-336, 2002.

14. Riggi N, Suvà ML, Suvà $\mathrm{D}$, Cironi L, Provero $\mathrm{P}$, Tercier $\mathrm{S}$, Joseph JM, Stehle JC, Baumer K, Kindler V and Stamenkovic I: EWS-FLI-1 expression triggers a Ewing's sarcoma initiation program in primary human mesenchymal stem cells. Cancer Res 68: 2176-2185, 2008

15. Liang X, Shi D, Yun J, Mao Y, Ouyang P, Su Z, Fu J, Hou J, Deng $\mathrm{W}$ and Xie F: Friend leukemia virus integration 1 expression has prognostic significance in nasopharyngeal carcinoma. Transl Oncol 7: 493-502, 2014

16. Jia D, Yan M, Wang X, Hao X, Liang L, Liu L, Kong H, He X, Li J and Yao M: Development of a highly metastatic model that reveals a crucial role of fibronectin in lung cancer cell migration and invasion. BMC Cancer 10: 364, 2010.

17. Asano Y, Stawski L, Hant F, Highland K, Silver R, Szalai G, Watson DK and Trojanowska M: Endothelial Fli1 deficiency impairs vascular homeostasis: A role in scleroderma vasculopathy. Am J Pathol 176: 1983-1998, 2010.
18. Zhang J, Guo H, Zhang H, Wang H, Qian G, Fan X, Hoffman AR, Hu JF and Ge S: Putative tumor suppressor miR-145 inhibits colon cancer cell growth by targeting oncogene Friend leukemia virus integration 1 gene. Cancer 117: 86-95, 2011.

19. Wu P, Liang J, Yu F, Zhou Z, Tang J and Li K: MiR-145 promotes osteosarcoma growth by reducing expression of the transcription factor friend leukemia virus integration 1. Oncotarget 7: 42241-42251, 2016.

20. Friedl $\mathrm{P}$ and Wolf $\mathrm{K}$ : Tumour-cell invasion and migration: Diversity and escape mechanisms. Nat Rev Cancer 3: 362-374, 2003.

21. Kleiner DE and Stetler-Stevenson WG: Matrix metalloproteinases and metastasis. Cancer Chemother Pharmacol 43 (Suppl): S42-S51, 1999.

22. Curran S and Murray GI: Matrix metalloproteinases in tumour invasion and metastasis. J Pathol 189: 300-308, 1999.

23. Kessenbrock K, Plaks V and Werb Z: Matrix metalloproteinases: Regulators of the tumor microenvironment. Cell 141: 52-67, 2010.

24. Itoh $T$, Tanioka M, Yoshida $H$, Yoshioka T, Nishimoto $H$ and Itohara S: Reduced angiogenesis and tumor progression in gelatinase A-deficient mice. Cancer Res 58: 1048-1051, 1998.

25. Ogasawara S, Yano H, Momosaki S, Nishida N, Takemoto Y, Kojiro $\mathrm{S}$ and Kojiro M: Expression of matrix metalloproteinases (MMPs) in cultured hepatocellular carcinoma (HCC) cells and surgically resected HCC tissues. Oncol Rep 13: 1043-1048, 2005.

26. Giannelli G, Bergamini C, Marinosci F, Fransvea E, Quaranta M, Lupo L, Schiraldi O and Antonaci S: Clinical role of MMP-2/ TIMP-2 imbalance in hepatocellular carcinoma. Int J Cancer 97: 425-431, 2002. 